\title{
De Novo Transcriptome Assembly for Venom Gland in Two Spe-Cies of Spiders (Sinopoda Pengi and Trichonephila Clavata)
}

\section{Li jun Ding}

Dali university

\section{Xiu mei Wu}

Dali university

Cheng gui Zhang

Dali university

\section{Peng fei Gao}

Dali university

\section{Yan Zhang}

Yunnan Provincial Academy of Science and Technology

\section{Yu Zhao}

Dali university

Zi zhong Yang ( $\nabla$ yangzizhong@Dali.edu.con )

Dali University https://orcid.org/0000-0002-2143-8226

\section{Research Article}

Keywords: spider venom gland, RNA-seq, encode venom, Sinopoda pengi, Trichonephila clavata

Posted Date: December 10th, 2021

DOI: https://doi.org/10.21203/rs.3.rs-1135110/v1

License: (c) (1) This work is licensed under a Creative Commons Attribution 4.0 International License. Read Full License 


\section{Abstract}

Natural molecules from spider venom are considered potential drugs for diseases including cancer and pain, as well as the development of new biological insecticides for agricultural use. During coevolution in the long-term predator-prey game, spiders have formed a huge molecular diversity of toxins. As of March 1 of 2021, a total of 49,243 spider species had been described, but studies of venom have been performed in only a few hundred of these species due to the difficulty of collecting venom. Two technologies have helped partially dealing with this limitation in the recent past: the screening of cDNA libraries constructed from venom gland mRNAs and the heterologous expression of the coded peptides for functional characterization. In this study, transcriptomic analysis was performed to describe the predicted toxins of Sinopoda pengi (hereafter S. pengi) and Trichonephila clavata (hereafter T. clavata). The Trinity assembly result in 163,418 transcripts, 114,127 unigene of $S$. pengi and 125,099 transcripts, 87,084 unigene of $T$. clavata. A total of 22 and 24 unigenes were identified which were predicted to inhibitor cysteine knot (ICK) toxins from S. pengi and T. clavata, respectively. In summary, molecular templates with potential application value in medical and biological fields were obtained by classifying and characterizing presumed venom components, which lays a foundation for the further study of venom.

\section{Introduction}

More than 100,000 species distributed across all major phyla of the animal kingdom have developed sophisticated machinery to produce poison or venom to defend themselves from predators, to fight against competitors, or to immobilize and digest prey [1-2]. Spiders are the seventh most diversified order of animals and the most diversified group of venomous predators in terms of the number of species, occupying most ecological niches [3]. Spider venoms are complex cocktails produced from the holocrine glands in the chelicerae. During coevolution in the long-term predator-prey game, venomous animals have formed a huge molecular diversity of animal toxins [2]. These toxin molecules usually act on the key physiological protein elements of the target organism, such as cell membrane receptors and ion channels (effectively distinguishing between different membrane receptors and ion channel subtypes) [4]. Toxins have become an important source of molecular tools for dissecting physiological processes [5] and are taken as lead molecules for the development of drugs targeting a range of conditions, such as chronic pain, cancer, stroke, and autoimmune disease [6].

Sinopoda pengi (Sparassidae; Fig. 1A) is a wandering spider with a widespread distribution in Yunnan, China, that lives on walls near houses or in rock cracks along roadsides. These spiders are ambush predators that do not spin webs but instead rely on their strong chelicerae and powerful venom to paralyze or kill their prey during hunting. They hunt at night, remaining quietly on walls waiting for prey to appear and then killing the prey when it passes by. They prey on nocturnal insects such as small beetles and crickets. In contrast, Trichonephila clavata (Araneidae; Fig. 1B) usually produces large, strong nets around hedges or bushes to facilitate prey capture, in addition to deploying its venom arsenal. These spiders are active during the day, and their web often contains the remains of butterflies, bees and other 
insects. These two species are relatively widespread spiders with different feeding habits, and their venom composition have not yet been studied with transcriptomic.

Spider venom received little attention for quite some time due to technological limitations and its low impact on human health until its enormous medical potential was discovered. As of March 1 of 2021, a total of 49,243 spider species had been described, but studies of venom have been performed in only a few hundred of these species [7]. Considering that individual venoms may contain hundreds of unique compounds and that the venoms of even comparatively well-studied lineages remain largely unexplored [2], animal venoms constitute an enormous unexplored natural library of bioactive compounds [8]. One of the reasons for the gap in venom knowledge resides in the difficulties imposed by the need to collect a large number of specimens from natural environments to "milk" their venom in large-enough quantities to allow the isolation of the less represented components [9]. The emergence and development of sequencing techniques has provided a solution to this awkward situation, and the toxin information available in the ArachnoServer and UniProt databases has increased rapidly in the last decade. Through transcriptome sequencing, we can determine the expression of the components associated with venom from just a few samples. Based on this approach, spider venom has been speculated to contain neurotoxins as well as other components, such as enzymes with serine proteinase, metalloprotease activity [10-12]. Here, only a few spiders are required to perform a detailed venom composition analysis via transcriptomic techniques.

\section{Materials And Methods}

\subsection{Biological sample and RNA extraction}

Specimens were collected in Dali, China $\left(25^{\circ} 40^{\prime} 41^{\prime \prime} \mathrm{N}, 100^{\circ} 8^{\prime} 59\right.$ "E; and $\left.25^{\circ} 40^{\prime} 52^{\prime \prime} \mathrm{N}, 100^{\circ} 10^{\prime} 20^{\prime \prime} \mathrm{E}\right)$ and were identified as S. pengi and T. clavata by Professor Zi-Zhong Yang of Dali University. The spiders were kept at the Yunnan Provincial Key Laboratory of Entomological Biopharmaceutical R\&D at room temperature under a natural light-dark cycle, fed mealworms on a weekly basis and provided with petri dishes as drinking vessels.

Venom glands were removed from the cephalothorax of each spider (Fig. 2) and total RNA was extracted from the venom glands of $5 \mathrm{~S}$. pengi and $5 \mathrm{~T}$. clavata using TRIzol, following manufacturer instructions, respectively (Invitrogen Life Technologies, USA). The concentration and purity of the RNA were checked with a Nanodrop 2000 (Thermo Fisher Scientific, USA). For RNA sequencing, RNA integrity was assessed by standard denaturing agarose gel electrophoresis.

\section{2. cDNA library preparation and Illumina sequencing}

Total RNA was used for the construction of RNA-seq libraries, and we performed paired-end sequencing on an Illumina NovaSeq 6000 system (Origin-gene Biopharm, China) following the vendor's recommended protocol. 
After adaptor clipping, the quality of the raw reads was assessed with FastQC v0.11.4 (www.bioinformatics.babraham.ac.uk/bugzilla/). The quantification of the raw data was performed using FastqStat.jar V1.0, followed by filtering to obtain clean data, which were analyzed using Cutadapt v1.16. (http://cutadapt.readthedocs.io/). All reads were submitted to NCBI with the login numbers SRR14235845 and SRR15356212 for S. pengi and T. clavata, respectively.

\subsection{Assembly and annotation}

The clean data were de novo assembled with Trinity v.2.6.6 using the standard protocol [13]. After the transcripts were assembled by Trinity, the functional annotation of these transcripts was performed. Before annotation, the open reading frame (ORF) prediction method of Trinity was used to predict the amino acid sequences of all assembled transcripts.

Annotation of de novo assembled transcripts was performed using Blastx

(http://blast.ncbi.nlm.nih.gov/Blast) based on the following databases: $\mathrm{Nr}$ (NCBI nonredundant protein sequences, https://www.ncbi.nlm.nih.gov/), Pfam (Protein family, http://pfam.sanger.ac.uk/), GO (Gene Ontology, http://www.geneontology.org), and KEGG (Kyoto Encyclopedia of Genes and Genomes, http://www.genome.jp/kegg/). Finally, gene expression levels were estimated in transcripts per kilobase million (TPM) values by Salmon v0.11.3.

\subsection{Bioinformatic analysis}

The unigenes used BLASTX searched against a toxin-related subdatabase of UniProt (https://www.uniprot.org/program/Toxins), best hits were defined using E-value cutoff of 1e-5 and selecting the best score. These unigenes were translated using the ORFfinder (https://www.ncbi.nlm.nih.gov/orffinder).

To remove potentially nontoxic orthologs, all candidate toxin unigenes were used as queries for the local BLASTX search (e-value $=1 \mathrm{e}-5$ ) against two databases: $(1)$ animal-reviewed proteins in the UniprotKB and (2) toxin protein database, as explained previously. Unigenes were filtered out from the candidate toxin gene dataset if the BLAST score of the best hit from the animal protein database was higher than the animal toxin database results [14].

The sequences which may encode for toxins were used for multiple sequence alignment and performed the alignments with Clustal Omega (version: 1.2.4), without a conserved region, were discarded. The signal peptide was predicted using the SignalP-5.0 program (http://www.cbs.dtu.dk/services/SignalP/); pro-peptides were determined by using ProP software (http://www.cbs.dtu.dk/services/ProP/).

\section{Results And Discussion}

\subsection{RNA extraction and RNA-Seq}


The total amounts of RNA obtained from fifteen adult female S. pengi and T. clavata, respectively. mRNA was isolated, enriched, fragmented and reverse transcribed into cDNA. Then, the cDNA libraries were sequenced using an Illumina NovaSeq 6000 system (LC Bio, China). The sequencing of the PE libraries produced 47,271,254 and 39,592,392 reads, respectively (Table S1).

\subsection{De novo transcriptome assembly and functional annotation}

There is no available reference genome for $S$. pengi or $T$. clavata, so Trinity needs to be used for de novo assembly. After assembly, we obtained 163,418 transcripts, 114,127 unigene of $S$. pengi and 125,099 transcripts, 87,084 unigene of $T$. clavata by using Trinity. After statistical analysis, transcripts were annotated in the NR, GO, KEGG, and Pfam databases (Supplementary Figs S1-3, Table S2-3).

\subsection{Possible toxins identified at the transcriptional level}

Detailed toxin information is provided in the supplementary materials (Supplementary Tables S4-5). Each transcript annotated as a toxin is discussed based on the existing literature, followed by a categorical description of the toxin.

\subsubsection{ICK-like spider venom peptides}

Cysteine-rich peptides are the best investigated venom components and are believed to exist in most spider venoms. They modulate a broad range of channels and receptors on the membranes of excitable cells (e.g., nerves and muscles) [15]. Ion channels on the membranes of excitable cells are responsible for proper signal transduction. Calcium channels are involved in neurotransmitter release from presynaptic cells, voltage-gated sodium channels enable action potential transmission along excitable cells, and voltage-gated potassium channels are crucial for restoring a resting state in depolarized cells [15]. Toxins interact with these targets to disrupt normal channel function, which can affect breathing or heart function, leading to symptoms such as convulsions, paralysis, and eventually death. Abundant ion channel toxins were identified by annotating the transcripts of S. pengi and T. clavata. The families of the predicted ICK toxins are described in detail below.

Group I (C-C-CC-C-C)

Group I contained three unigenes in T. clavata, two of which (DN1625_c0_g1, DN16263_c0_g1) were similar to Mu-Sparatoxin Hv2 (UniProt A0A088BP94) and one (DN5056_c0_g1) was similar to KappaSparatoxin Hv1a (UniProt P58425) (Fig. 3A). These reference toxins belong to the neurotoxin 10 (Hwtx-1) family. Mu-sparatoxin-Hv2 from Heteropoda venatoria, is insecticidal toxin potently and irreversibly blocks $\mathrm{Na}_{\mathrm{V}}$ channels in cockroach dorsal unpaired median (DUM) neurons $\left(\mathrm{IC}_{50}=833.7 \mathrm{nM}\right)$ [16]. $\mathrm{Na}_{\mathrm{V}}$ channels are crucial in the generation and transmission of action potentials in the central nervous system, peripheral nervous system, heart, smooth muscle and skeletal muscle, so the function of $\mathrm{Na}_{\mathrm{V}}$ channels is crucial, and they are targets of many toxins. There are nine a subtypes, known as $\mathrm{Na}_{\mathrm{v}} 1.1$ - 
$\mathrm{Na}_{\mathrm{V}} 1.9$ in mammals, each with different tissue distributions and functions [17]. The reference toxin Kappa-Sparatoxin $\mathrm{Hv} 1 \mathrm{a}$ is a potassium and calcium channel blocker. $\mathrm{K}_{\mathrm{V}}$ channels play an important role in human physiology, including the regulation of neurotransmitter release, heart rate, insulin secretion, nerve cell secretion, skeletal muscle contraction, etc. Numerous channelopathies arising from mutations in these channels or from autoimmune attack on the channels have been characterized [18-19]. Potassium channels are the target of a variety of toxic animal toxins, and the potassium channel inhibitors found in spider venom thus far mainly act on $\mathrm{K}_{\mathrm{v}} 1, \mathrm{~K}_{\mathrm{V}} 2$ and $\mathrm{K}_{\mathrm{v}} 4$ channels, although a few act on other channels, such as $\mathrm{K}_{\mathrm{V}} 3, \mathrm{~K}_{\mathrm{V}} 7$ and $\mathrm{K}_{\mathrm{V}} 11$ channels [20]. In $S$. pengi, Group I contained 9 unigenes (Fig. 3B), two of them showed homology with kappa-sparatoxin-Hv1a and seven were aligned to Musparatoxin-Hv2.

\section{Group II (C-C-CC-CXC-CXC)}

In T. clavata, Group II included 5 sequences (Fig. 4A), correspond to U3-lycotoxin-Ls1b (UniProt B6DCQ7), LSTX-D6 (UniProt B6DCU5), U1-lycotoxin-LS1B (UniProt B6DCK2), U19-Lycotoxin-LS1B (UniProt B6DD58) from Lycosa singoriensis and U8-agatoxin Ao1a (UniProt Q5Y4U4) from Agelena orientalis, respectively. The ion channel activity of these reference peptides is not known.

In S. pengi, Group II is composed of 5 sequences (Fig. 4B). One of them resembled Omega-SegestritoxinSf1a (UniProt P58605) from Segestria florentina, another resembled U10-Ctenitoxin-Pr1a (UniProt P84000) and the other three resemble U6-Lycotoxin-LS1g (UniProt B6DCV8) from Lycosa singoriensis. Omega-Segestritoxin-Sf1a is potent and selective blocker of N-type voltage-gated calcium channels (Cav2.2/CACNA1B), also blocks vertebrate Cav2.1/CACNA1A (P/Q-type) and Cav1.2/CACNA1C (L-type) channels at very high concentration (2 micromolar) [21].

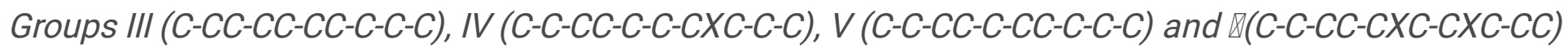

In T. clavata, Group III contained only one sequence (DN18027_c0_g1) (Fig. 5), which had 66.3\% identity with Omega-agatoxin-1A from Agelenopsis aperta. Omega-agatoxins are antagonists of voltage-gated calcium channels, subtypes of omega-agatoxins are distinguished as type I (omega-Aga-IA, omega-AgaIB, and omega-Aga-IC), type II (omega-Aga-IIA and omega-Aga-IIB), and type III (omega-Aga-IIIA and omega-Aga-IIIB). All except type III toxins block calcium channels in insect motor nerve terminals and in neuronal cell bodies at nanomolar concentrations [22]. No sequence from $S$. pengi in this family.

In T. clavata, group IV contained 9 unigenes (Fig. 6A), 5 of which displayed similarity to toxin CSTX-20 from Cupiennius salei (UniProt B3EWT5). One of which presented identity to U19-ctenitoxin-Pn1a (UniProt P83997) from Phoneutria nigriventer. Three other sequences were similar to U3-aranetoxin-Ce1a3 (UniProt Q8MTX1) from Caerostris extrusa. In S. pengi, one sequence is similar to U33-theraphotoxin$\mathrm{Cg} 1 \mathrm{~b}$ (UniProt B1P1J0) from Chilobrachys guangxiensis and one to U8-theraphotoxin-Hhn1d (UniProt D2Y2E4) from Cyriopagopus hainanus, the other 5 unigenes correspond to CSTX-20 (Fig. 6B). 
In T. clavata, group V consisted of 4 unigenes (Fig. 7), two of them showed homology with U20-lycotoxinLs1c (UniProt B6DD62) from Lycosa singoriensis. The other two sequences were similar to U15-lycotoxinLs1a (UniProt B6DD43) from Lycosa singoriensis and U7-agatoxin-Ao1a (UniProt Q5Y4V9) from Agelena orientalis, respectively. No sequence from $S$. pengi in this family.

In S. pengi, group $₫$ contained only one sequence (Fig. 8), the sequence had homology to U16-lycotoxinLs1b (UniProt B6DD53C) from Lycosa singoriensis. No sequence from T. clavata in this family.

\section{Group $\otimes(C-C-C-C C-C X C-C X C-C-C-C)$}

In T. clavata, Group $\square$ was represented by only two sequences (DN21003_c0_g1, DN51867_c0_g1), which had identity with U9-agatoxin-Ao1a (UniProt Q5Y4U3) from Agelena orientalis. No sequence from S. pengi in this family (Fig. 9).

\subsubsection{Enzymes}

Enzymes are very common components of the venom of poisonous animals such as poisonous snakes, scorpions and centipedes. Proteases are enzymes that hydrolyze the amide bonds of the peptide units of polypeptides and proteins. The overall purpose of such an enzyme arsenal co-injected with toxins into a prey's tissue seems clear: by destroying the barriers imposed by the extracellular matrix and cell membranes, the toxins can quickly reach their targets [23]. Additionally, the proteolytic activity of some of these enzymes facilitates subsequent preoral digestion. As proteins play important roles in the maintenance of homeostasis, proteases are vital regulators of physiological processes. Many pathological conditions of humans and animals have been linked to the malfunctioning of this category of enzymes; as a result, they are seen as attractive targets for drug discovery [24].

Unigenes related to metalloproteinases were found in S. pengi and T. clavata. The role of metalloproteinases in venoms is not fully understood, but it may be a diffusion factor promoting the diffusion of other venoms. It may be involved in proteolysis of other venomous toxins, or it may aid extraoral digestion of prey [25].

In S. pengi, we found unigenes may encoding acetylcholinesterase. Acetylcholine-mediated neurotransmission is fundamental for nervous system function. Acetylcholinesterase (AChE) hydrolyses and inactivates acetylcholine, thereby regulating the concentration of the transmitter at the synapse [26]. In venom, the toxic role of this enzyme is unclear; it could reduce musculatory control by rapidly hydrolyzing acetylcholine, or it works synergistically with alkaline phosphatase to paralyze prey through hypotension.

\section{Conclusion}

Through RNA-seq, we performed a detailed analysis of all predicted toxins of S. pengiand T. clavata. The results can serve as a reference for other spider toxicological studies and provide valuable molecular templates for research and therapeutic applications. 


\section{Declarations}

\section{Acknowledgments}

This work was funded by The Special Program of Science and Technology of Yunnan Province (202002AA100007), Yunnan Education Department (2021Y401).

Conflicts of Interest The authors have declared that no competing interests exist.

Ethical approval All applicable international, national, and/or institutional guidelines for the care and use of animals were followed.

\section{References}

1. Calvete, JJ (2017) Venomics: integrative venom proteomics and beyond. Biochem. J. 474, 611-634. https://doi.org/10.1042/BCJ20160577

2. Schendel, V, Rash, LD, Jenner, RA, Undheim, EAB (2019) The Diversity of Venom: The Importance of Behavior and Venom System Morphology in Understanding Its Ecology and Evolution. Toxins (Basel) 11, 666-687. https://doi.org/10.3390/toxins 11110666

3. Coddington, JA, Levi, HW (1991) Systematics and evolution of spiders (Araneae). Annu. Rev. Ecol. Syst. 22, 565-592. https://doi.org/10.1146/annurev.es.22.110191.003025

4. Sunagar, K, Casewell, NR, Varma, S, Kolla, R, Antunes, A, Moran, Y (2016) Deadly Innovations: Unraveling the Molecular Evolution of Animal Venoms. Springer Netherlands. https://doi.org/10.1007/978-94-007-6416-3_27

5. Akef, HM (2018) Anticancer, antimicrobial, and analgesic activities of spider venoms. Toxicology Research 7, 381-395. https://doi.org/10.1039/c8tx00022k

6. Peigneur, S, Tytgat, J (2018) Toxins in Drug Discovery and Pharmacology. Toxins (Basel) 10, 126129. https://doi.org/10.3390/toxins 10030126

7. Pineda, SS, Chaumeil, P-A, Kunert, A, Kaas, Q, Thang, MWC, Li, L, Nuhn, M, Herzig, V, Saez, NJ, Cristofori-Armstrong, B, Anangi, R, Senff, S, Gorse, D, King, GF (2017) ArachnoServer 3.0: an online resource for automated discovery, analysis and annotation of spider toxins. Bioinformatics 34 , 1074-1076. https://doi.org/10.1093/bioinformatics/btx661

8. Herzig, V, King, GF, Undheim, EAB (2019) Can we resolve the taxonomic bias in spider venom research? Toxicon 1, 100005. https://doi.org/10.1016/j.toxcx.2018.100005

9. Romero-Gutierrez, T, Peguero-Sanchez, E, Batista, CVF, Ortiz, E, Cevallos, MA, Possani, LD (2017) A Deeper Examination of Thorellius atrox Scorpion Venom Components with Omic Techonologies. Toxins (Basel) 9, 399-421. https://doi.org/10.3390/toxins9120399

10. Tang, X, Zhang, Y-Q, Hu, W-J, Xu, D-H, Tao, H, Yang, X-X, Li, Y, Jiang, L-P, Liang, S-P (2010) Molecular Diversification of Peptide Toxins from the Tarantula Haplopelma hainanum (Ornithoctonus hainana) 
Venom Based on Transcriptomic, Peptidomic, and Genomic Analyses. J Proteome Res 9, 25502564. https://doi.org/10.1021/pr1000016

11. Diego-García, E, Cologna, CT, Cassoli, JS, Corzo, G (2015) Spider Transcriptomes from Venom Glands: Molecular Diversity of Ion Channel Toxins and Antimicrobial Peptide Transcripts, Spider Venoms. https://doi.org/10.1007/978-94-007-6646-4_17-1

12. Paiva, ALB, Mudadu, MA, Pereira, EHT, Marri, CA, Guerra-Duarte, C, Diniz, MRV (2019) Transcriptome analysis of the spider Phoneutria pertyi venom glands reveals novel venom components for the genus Phoneutria. Toxicon 163, 59-69. https://doi.org/10.1016/j.toxicon.2019.03.014

13. Grabherr, MG, Haas, BJ, Yassour, M, Levin, JZ, Thompson, DA, Amit, I, Adiconis, X, Fan, L, Raychowdhury, R, Zeng, Q, Chen, Z, Mauceli, E, Hacohen, N, Gnirke, A, Rhind, N, Palma, Fd, Birren, BW, Nusbaum, C, Lindblad-Toh, K, Friedman, N, Regev, A (2011) Full-length transcriptome assembly from RNA-Seq data without a reference genome. Nat Biotechnol 29, 644-652. https://doi.org/10.1038/nbt.1883

14. Mitpuangchon, N, Nualcharoen, K, Boonrotpong, S, Engsontia, P (2021) Identification of Novel Toxin Genes from the Stinging Nettle Caterpillar Parasa lepida (Cramer, 1799): Insights into the Evolution of Lepidoptera Toxins. Insects 12. https://doi.org/10.3390/insects12050396

15. Langenegger, N, Nentwig, W, Kuhn-Nentwig, L (2019) Spider Venom: Components, Modes of Action, and Novel Strategies in Transcriptomic and Proteomic Analyses. Toxins 11, 611-654. https://doi.org/10.3390/toxins11100611

16. Xiao, Z, Zhang, Y-X, Zeng, J, Liang, S-P, Tang, C, Liu, Z-H (2018) Purification and Characterization of a Novel Insecticidal Toxin, $\mu$-sparatoxin-Hv2, from the Venom of the Spider Heteropoda venatoria. Toxins (Basel) 10, 233-244. https://doi.org/10.3390/toxins10060233

17. Israel, MR, Tay, B, Deuis, JR, Vetter, I (2017) Sodium Channels and Venom Peptide Pharmacology. Adv Pharmacol 79, 67-116. https://doi.org/10.1016/bs.apha.2017.01.004

18. Norton, RS, Chandy, KG (2017) Venom-derived peptide inhibitors of voltage-gated potassium channels. Neuropharmacology 127, 124-138. https://doi.org/10.1016/j.neuropharm.2017.07.002

19. Tajti, G, Wai, DCC, Panyi, G, Norton, RS (2020) The voltage-gated potassium channel $K_{V} 1.3$ as a therapeutic target for venom-derived peptides. Biochem. Pharmacol. 181, 114146. https://doi.org/10.1016/j.bcp.2020.114146

20. Tabakmakher, VM, Krylov, NA, Kuzmenkov, Al, Efremov, RG, Vassilevski, AA (2019) Kalium 2.0, a comprehensive database of polypeptide ligands of potassium channels. Sci Data 6, 73-80. https://doi.org/10.1038/s41597-019-0074-x

21. Newcomb, R, Palma, A, Fox, J, Gaur, , S. , L, K. , (1995) SNX-325, a novel calcium antagonist from the spider Segestria florentina. Biochem 34, 8341-8347. https://doi.org/10.1021/bi00026a015

22. Bindokas, VP, Venema, VJ, Adams, ME (1991) Differential antagonism of transmitter release by subtypes of omega-agatoxins. J Neurophysiol 66, 590. https://doi.org/10.1016/01655728(91)90061-B 
23. Kuhn-Nentwig, L, Stöcklin, R, Nentwig, W (2011) Venom Composition and Strategies in Spiders: Is Everything Possible? Adv. Insect Physiol. 40, 1-86. https://doi.org/10.1016/b978-0-12-3876683.00001-5

24. Agbowuro, AA, Huston, WM, Gamble, AB, Tyndall, JDA (2018) Proteases and protease inhibitors in infectious diseases. Med Res Rev 38, 1295-1331. https://doi.org/10.1002/med.21475

25. Silveira., RB, Wille, AC, Chaim, OM, Appel, MH, Silva, DT, Franco, CR, Toma, L, Mangili, OC, Gremski, W, Dietrich, CP, Nader, HB, Veiga, SS (2007) Identification, cloning, expression and functional characterization of an astacin-like metalloprotease toxin from Loxosceles intermedia (brown spider) venom. Biochem. J. 406, 355-363. https://doi.org/10.1042/BJ20070363

26. Hermona Soreq, Seidman, S (2001) Acetylcholinesterase-new roles for an old actor. Perspectives (Montclair) 2, 294-302. https://doi.org/10.1038/35067589

\section{Figures}
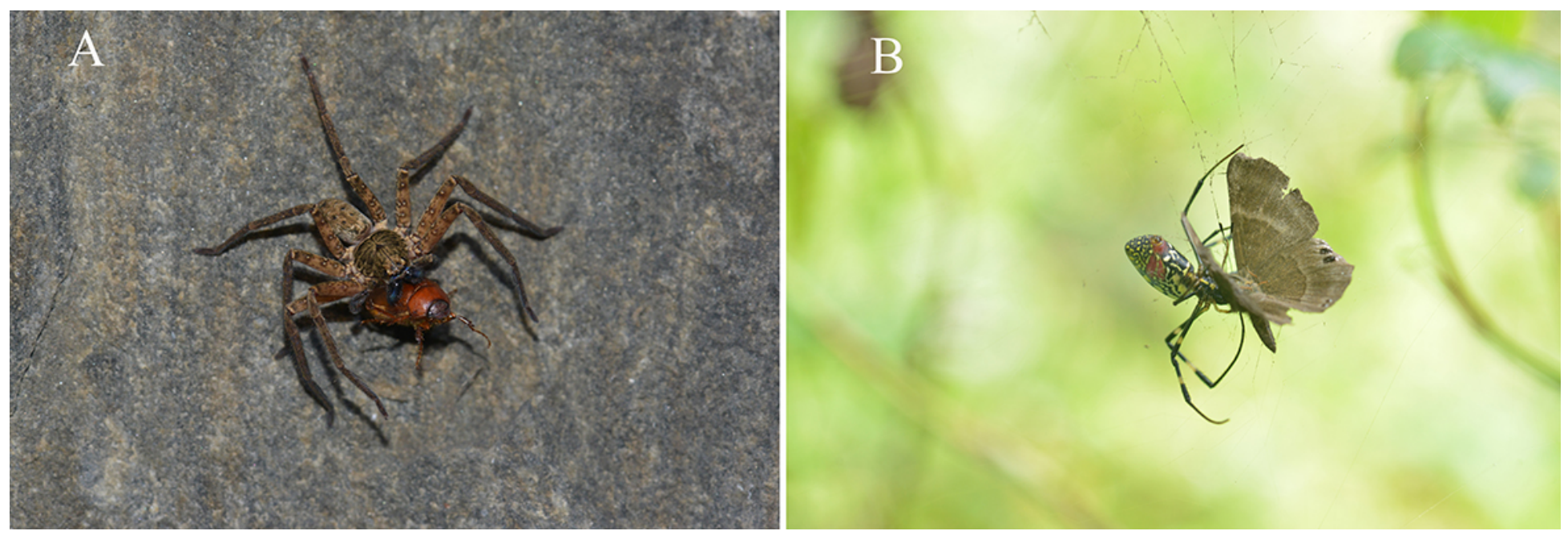

Figure 1

Spiders and their prey. A. S. pengi hunting a cockchafer, B. T. clavata preying on a butterfly.

\section{Figure 2}

Isolation of spider venom glands. A1. Dissecting the venom glands of T. clavata, A2 venom glands of T. clavata, B1. Dissecting the venom glands of S. pengi, B2. venom glands of S. pengi. 
T.clavata_DN1625_c0_g1

T.clavata_DN5056_cO_g1

sp|A0A088BP94|Mu-sparatoxin-Hv2

sp|P58425|Kappa-sparatoxin-Hv1a

T.clavata_DN16263_c0_g1

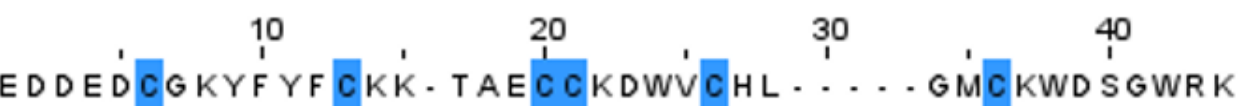
... SCGTIWHYCGTDQSECCEGWKCSR . . . QLCKYVIDWGK . DDDCGKLFADCTS - DSDCCENWVCSKTGFVKN ICKYNFG... ... DCGTIWHYCGTDQSECCEGWKCSR . . . QL CKYVIDW. . - DDQYCSYPHSPCDK. YPCRCPGYECYD... GMCGEIRRRD.

3B

TRINITY_DN17662_CO_g1 TRINITY_DN33845_c0_g1 sp|P58425|Kappa-sparatoxin-Hv1a TRINITY_DN883_cO_g2

TRINITY_DN9047_c0_g1 TRINITY_DN23341_c1_g1 TRINITY_DN35302_cO_g1 TRINITY_DN41330_cO_g1 TRINITY_DN52140_cO_g1 TRINITY_DN68838_c1_g1 sp|A0A088BP94|MU-sparatoxin-Hv2

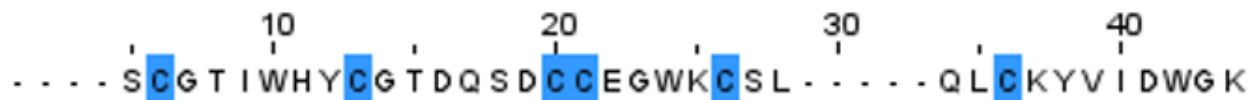
... SCGT IWHYCGTDQSECCEGWKCSR ....QLCKYVIDWGK ... DCGT IWHYCG TDQSECCEGWKCSR . . . QL CKYVIDW. . - QDDDCSDLYGSCRE . L LPCCPSYVCLG . . . N ICVITVR . . - DDEYCSYPEF SCRR - SENCCPGFMCYD . . . NMCTM . . . . - DDEYCSYPEF SCRR - SENCCPGYMCYN . . . Y YMCTL I . . . EDDEDCVKYFYFCKK. TAECCKVWVCHL . . . GMCKWDSGWRK .. TDDCGWLLSSCKS - SKTCCENWVCSK..... GLCKYRIG... EDDEDCGKYFYFCKK. TAECCKDWVCHL ..... GMCKW..... ... VCGTHFSLCDK. ESHCCEGYTCILW. . . . . CRKNL - W. . . DDDCGKLFADCTS. DSDCCENWVCSKTGFVKN I CKYNFG...

\section{Figure 3}

Alignments of cysteine-rich peptide toxins mature sequences from group $\mathbb{Q}$ (C-C-CC-C-C) in T. clavata. All alignment was performed with Clustal OMEGA. Conserved cysteines are marked in blue.

$4 \mathrm{~A}$

T.clavata_DN2914_co_g1 sp|B60CQ7|Uअ ycotoxin-Ls $1 b$ T.clavata_DN5074_c0_g1 sp|B 60 CU5|LSTX-D6

T.clavata_DN17357_c0_g1 sp|B60CK2|U1-1ycotoxin-Ls1b T.clavata_DN48781_co_g2 sp| $|860058| U 19-1 y c o t o x i n-L s 1 b$ T.clavata_DN1126_co_g1

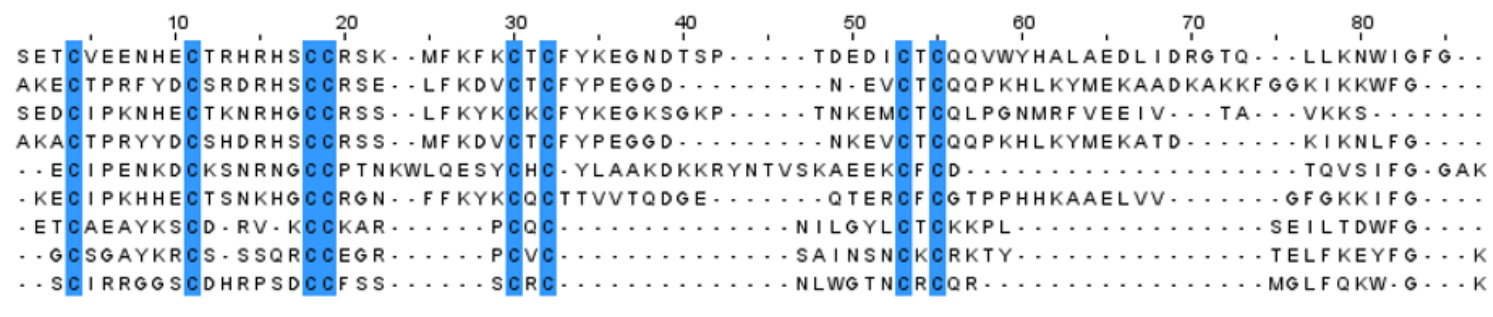

4B

TRINITY_DN1_c0_g1 sp|P58605|Omega-segestritoxin-Sf1a TRINITY_DN919_c0_g2 TRINITY_DN14378_cO_g3 TRINITY_DN26733_c0_g1 sp|B 60 CV8|U6-1ycotoxin-Ls1g TRINITY_DN896_c0_g1 sp|P84000|U10-ctenitoxin-Pr1a

\begin{abstract}
. 20

$30 \quad 40$ 50 60

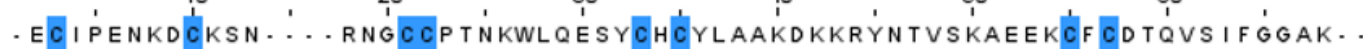
GSCIESGKSCTHSRSMKNGLCCPKS . . . . RCNCRQIQHRH - DY. . LGKRKYSCRCS . . . . . . . ESCSPQENRCFKE....GPKCCGGF .... DCRCYT . ... PIVNGVKEEVVCWCNEPNVLYEYAYKV - QC I KLETECTKN.... KGNCCGGH...... GCRCYR .... KYVNGVKAEVRCWCIENDVTYEPRIEA . QCPHIEQECTIK... KGI C CNEE..... ECRCYR..... KYINDVEGGVRCWCTNVDETYLPV... - ACI PLEKECTKT... PGNCCSGL..... KCDCYR..... RFEQGVAKGVQCWCIEKDVTYKGV..

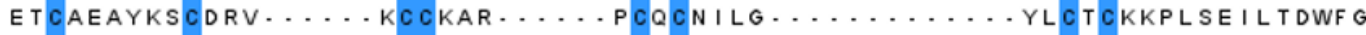

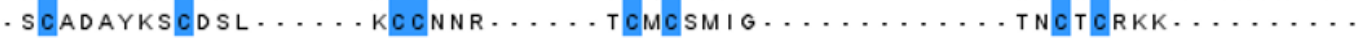


Alignments of cysteine-rich peptide toxins mature sequences from group (C-C-CC-CXC-CXC) in S. pengi.

T.clavata_DN18027_co_g1 sp|P15969|Omega-agatoxin-1A $\begin{array}{cccccccc}10 & 20 & 30 & 40 & 50 & 60 & 70 \\ 1 & 1 & 1 & 1 & 1\end{array}$

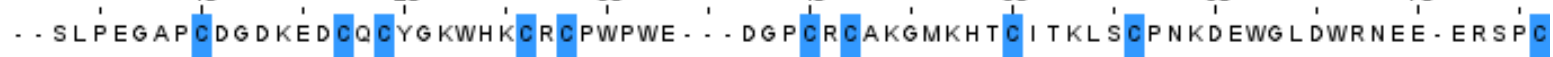
AKALPPGSVCDGNESDCKCYGKWHKCRCPWKWHF TGEGPCTCEKGMKHTCI TKLHCPNKAEWGLDWRSEESERSPC

\section{Figure 5}

Alignments of cysteine-rich peptide toxins mature sequences from groups $₫$ in $T$. clavata.

\section{Figure 6}

Alignments of cysteine-rich peptide toxins mature sequences from group III (C-CC-CC-CC-C-C-C) in T. clavata (6A) and S. pengi (6B).

T.clavata_DN51959_c0_g1 T.clavata_DN58023_cO_g1 sp|B60043|U15-ycotoxin-Ls1a T.clavata_DN2521_cO_g1 $s p|B 60061| \cup 20$-1ycotoxin- $L s 1 b$ T.clavata_DN11433_cO_g1 sp|Q5Y4V9|U7-agatoxin-Ao 1a

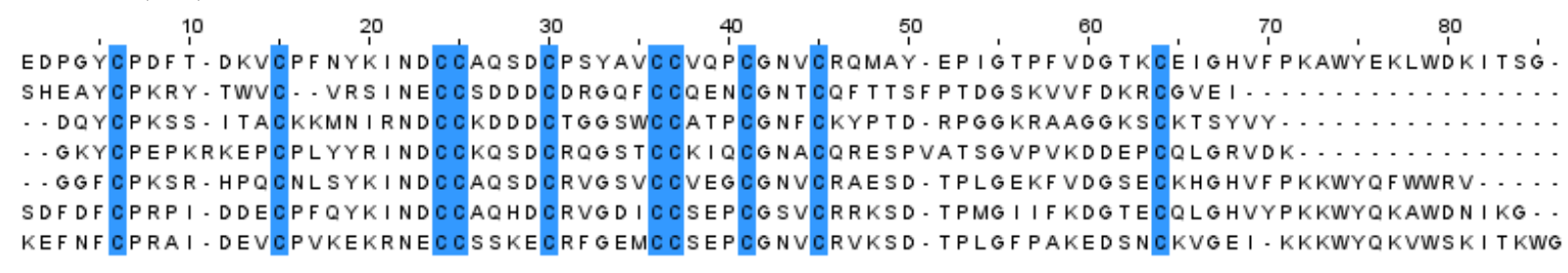

\section{Figure 7}

Alignments of cysteine-rich peptide toxins mature sequences from group IV (C-C-CC-C-C-CXC-C-C) in T. clavata.

\section{Figure 8}

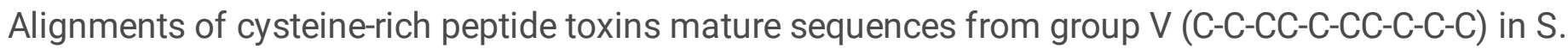
pengi.

T.clavata_DN21003_cO_g1 T.clavata_DN51867_c0_g1 sp|Q5Y4U3|U9-agatoxin-Ao 1a

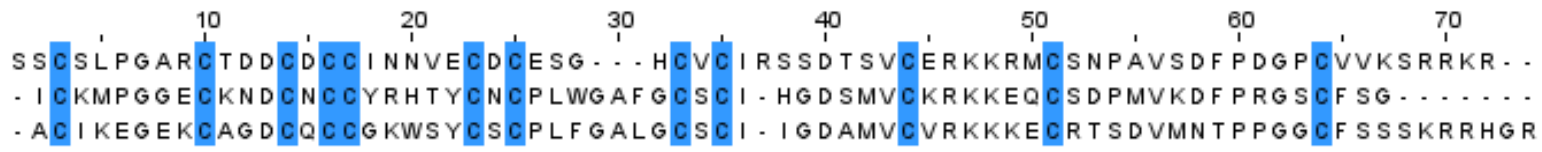

\section{Figure 9}

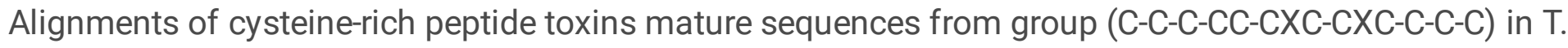
clavata.

\section{Supplementary Files}


This is a list of supplementary files associated with this preprint. Click to download.

- TableS1Assemblystatistics.xlsx

- TableS2unigeneannotationofS.pengi.csv

- TableS3unigeneannotationofT.clavata.csv

- TableS4ToxinsinformationofT.clavata.xlsx

- TableS5toxinsinformationofS.pengi.xlsx 